Preprint of the paper:

"Computer Analysis of Earthing Systems in Horizontally or Vertically Layered Soils"

I. Colominas, J. Gómez-Calviño, F. Navarrina, M. Casteleiro (2001)

Electric Power Systems Research, 59, 149-156.

http://caminos.udc.es/gmni 


\title{
COMPUTER ANALYSIS OF EARTHING SYSTEMS IN HORIZONTALLY OR VERTICALLY LAYERED SOILS
}

\author{
I. Colominas, J. Gómez-Calviño, F. Navarrina, and M. Casteleiro \\ Applied Mathematics Dept., Civil Engineering School \\ Universidad de La Coruña, Campus de Elviña, 15192 SPAIN \\ colominas@iccp.udc.es
}

\begin{abstract}
The main objectives of grounding systems are to guarantee the personal safety, the equipment protection and the continuity of the power supply. To attain these goals, the potential distribution on the earth surface and the equivalent resistance of the earthing system have to be determined in a reliable an efficient way. In this paper, we present a numerical technique based on the Boundary Element Method for grounding analysis of horizontally and vertically layered soils. The feasibility of the proposed method is demonstrated by means of its application to a real grounding grid considering different types of soil models.
\end{abstract}

\section{Keywords}

Grounding Analysis, Fault Protection, Layered Soils, BEM.

\section{Introduction}

Designing safe grounding systems for large electrical installations has been a challenging problem since the early days of the industrial use of electricity. Thus, the most important parameters that are required to design a safe grounding system are the potential distribution on earth surface when a fault current is derived into the soil and the equivalent resistance of the system. As it is known, a grounding system comprises all the interconnected grounding facilities of an specific area, being the grounding grid its main element. In general, most of grounding grids of electrical substations consist of a mesh of interconnected cylindrical conductors, horizontally buried, and supplemented by vertically thrusted ground rods in certain places of the substation site $[1,2,3]$

Equations governing the electrical current dissipation into the soil through a grounded electrode are well-known and can be stated from Maxwell's Electromagnetic Theory. Nevertheless, their application and resolution in practical cases for grounding grids of large installations present some troubles (obviously no analytical solutions can be obtained in a real problem).

Since the sixties, several methods for the grounding analysis and design of electrical substations have been proposed. These methods (generally based on professional practice, semi-empirical works, experimental data obtained from scale model assays, or intuitive ideas) represented an important improvement in the grounding analysis field. However, some problems (such as large computational requirements, unrealistic results when segmentation of conductors is increased, and uncertainty in the margin of error) have been reported $[1,4,5,6]$.

On the other hand, widespread numerical techniques commonly used in other fields of Engineering (such as the FEM and the FDM) are precluded in grounding analysis. The reason is that the required discretization of the soil would imply an unacceptable computational cost due to the characteristic geometry of these systems: a mesh of interconnected bare 
conductors with a small ratio diameter/length. In the last years the authors have developed a numerical formulation based on the Boundary Element Method for the analysis of grounding systems embedded in uniform soils [7, 8, 9]. This approach has been implemented in a CAD system [10] that currently allows to analyze real grounding grids in real-time in personal computers. Furthermore, this formulation has been extended for the grounding analysis in stratified soils [11]. In this paper, we present a summary of the proposed method, and its application to a real system with several soil models.

\section{Mathematical model of the problem}

\subsection{Governing equations of the problem}

As we have exposed in the previous section, the problem can be stated by means of Maxwell's equations. Thus, restricting the analysis to the electrokinetic steady-state response and neglecting the inner resistivity of the earthing conductors (potential is assumed constant on the electrode surface), the $3 \mathrm{D}$ problem can be written as

$$
\begin{gathered}
\operatorname{div}(\boldsymbol{\sigma})=0, \quad \boldsymbol{\sigma}=-\boldsymbol{\gamma} \operatorname{grad}(V) \text { in } E \\
\boldsymbol{\sigma}^{t} \boldsymbol{n}_{E}=0 \text { in } \Gamma_{E} ; V=V_{\Gamma} \text { in } \Gamma ; V \rightarrow 0, \text { if }|\boldsymbol{x}| \rightarrow \infty
\end{gathered}
$$

being $E$ the earth, $\boldsymbol{\gamma}$ its conductivity tensor, $\Gamma_{E}$ the earth surface, $\boldsymbol{n}_{E}$ its normal exterior unit field and $\Gamma$ the electrode surface [9]. Therefore, when the electrode attains a voltage $V_{\Gamma}$ (Ground Potential Rise, or GPR) relative to a distant grounding point, the solution to problem (1) gives the potential $V$ and the current density $\boldsymbol{\sigma}$ at an arbitrary point $\boldsymbol{x}$ [9]. Consequently, it is possible to obtain the potential distribution on the earth surface, the total surge current and the equivalent resistance of the grounding system [1,9] by solving problem (1). Since $V$ and $\boldsymbol{\sigma}$ are proportional to the GPR value, the normalized boundary condition $V_{\Gamma}=1$ is not restrictive at all, and it will be used from here on [9].

In most of the proposed methods for grounding analysis, the soil is considered homogeneous and isotropic, being the conductivity tensor $\boldsymbol{\gamma}$ substituted by an experimentally obtained scalar value $\gamma$. This hypothesis does not introduce significant errors if the soil is essentially uniform in all directions in the vicinity of the substation site, and it can be used without loss of accuracy if the soil resistivity changes slightly with depth [1]. Nevertheless, safety parameters involved in the grounding design may significantly vary if the electrical properties of the soil change in the surroundings of the electrical installation (for example, due to changes of the material nature, or the humidity of the soil). Thus, it seems advisable to develop more advanced soil models. Clearly, to consider all the variations of the soil conductivity would never be affordable neither from the economical nor from the technical point of view. For this reason, a frequently proposed more practical soil model consists of assuming the soil stratified in a number of horizontal layers, defined by their thicknesses and their apparent scalar conductivities that must be experimentally obtained. In fact, it is widely accepted that two-layer soil models should be sufficient to obtain safe designs of grounding systems in most practical cases [1].

Then, in a stratified soil model formed by $C$ layers with different conductivities and being the grounded electrode buried in the layer $b,(1)$ can be written in terms of the following Neumann exterior problem

$$
\begin{gathered}
\operatorname{div}\left(\boldsymbol{\sigma}_{c}\right)=0, \boldsymbol{\sigma}_{c}=-\gamma_{c} \operatorname{grad}\left(V_{c}\right) \text { in } E_{c}, 1 \leq c \leq C ; \\
\boldsymbol{\sigma}_{1}^{t} \boldsymbol{n}_{E}=0 \text { in } \Gamma_{E} ; V_{b}=1 \text { in } \Gamma ; \\
V_{c} \rightarrow 0 \text { if }|\boldsymbol{x}| \rightarrow \infty, 1 \leq c \leq C-1 ; \\
\boldsymbol{\sigma}_{c}^{t} \boldsymbol{n}_{c}=\boldsymbol{\sigma}_{c+1}^{t} \boldsymbol{n}_{c} \text { in } \Gamma_{c}, 1 \leq c \leq C-1 ;
\end{gathered}
$$

being $E_{c}$ each one of the soil layers, $\gamma_{c}$ its conductivity, $V_{c}$ the potential at an arbitrary point of $E_{c}, \boldsymbol{\sigma}_{c}$ its corresponding current density, $\Gamma_{c}$ the interface between layers $E_{c}$ and $E_{c+1}$, and $\boldsymbol{n}_{c}$ the normal unit field to $\Gamma_{c}$ [11].

\subsection{Integral expression for potential $V$}

As we have exposed in the introduction, the specific geometry of the grounding grids in practice makes difficult the use of standard numerical methods, since discretization of the $3 \mathrm{D}$ domains $E_{c}$ would involve a completely out of range computing effort. Because of this, we have turned our attention to other numerical techniques that only require the discretization of the boundary [9].

It is known that the surroundings of a substation site are levelled and regularized during its constructive phase. These facts can be taken into account in the soil model, so that earth surface $\Gamma_{E}$ and interfaces $\Gamma_{c}$ between layers can be 
assumed horizontal. Now, the application of the "method of images" and Green's Identity to problem (2) yields the following integral expression [11] for potential $V_{c}\left(\boldsymbol{x}_{c}\right)$ at $\boldsymbol{x}_{c} \in E_{c}$ :

$$
V_{c}\left(\boldsymbol{x}_{\boldsymbol{c}}\right)=\frac{1}{4 \pi \gamma_{b}} \iint_{\boldsymbol{\xi} \in \Gamma} k_{b c}\left(\boldsymbol{x}_{c}, \boldsymbol{\xi}\right) \sigma(\boldsymbol{\xi}) d \Gamma, \forall \boldsymbol{x}_{c} \in E_{c}
$$

where $\sigma(\boldsymbol{\xi})$ is the leakage current density $\left(\sigma=\boldsymbol{\sigma}^{t} \boldsymbol{n}\right.$, being $\boldsymbol{n}$ the normal exterior unit field to $\left.\Gamma\right)$ at any point $\boldsymbol{\xi}$ of the electrode surface $\Gamma \subset E_{b}$.

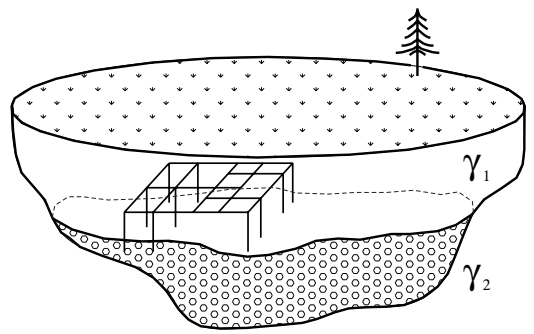

Figure 1. Scheme of horizontally layered soil model

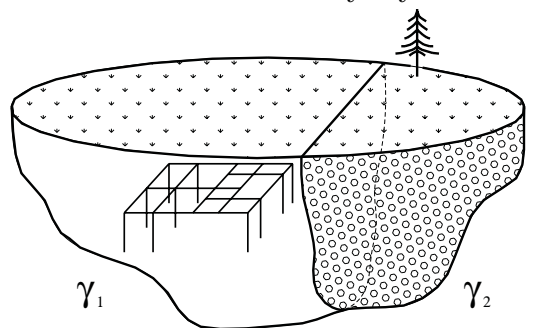

Figure 2. Scheme of vertically layered soil model

In general, the integral kernel $k_{b c}\left(\boldsymbol{x}_{c}, \boldsymbol{\xi}\right)$ is formed by series of terms corresponding to the resultant images obtained when Neumann exterior problem (2) is transformed into a Dirichlet one [11, 12]. Depending on the type of the soil model being used, these series can have an infinite or a finite number of terms (e.g., for a uniform soil model the series only contain two summands). Each one of these terms depends on the conductivities of the layers and on the inverse of the distances from $\boldsymbol{x}$ to $\boldsymbol{\xi}$ and from $\boldsymbol{x}$ to all the images of $\boldsymbol{\xi}$ with respect to the earth surface $\Gamma_{E}$ and with respect to all the interfaces between layers [11].

Although the generation of electrical images in a general case with an arbitrary number of layers is a conceptually simple well-known process, obtaining the final expressions of the integral kernels $k_{b c}\left(\boldsymbol{x}_{c}, \boldsymbol{\xi}\right)$ may require extensive analytical work, the evaluation of which may imply a high computational effort in practice. The examples presented in this paper correspond to cases with uniform or two layered soil models. From now on we will only refer to these cases. Figures 1 and 2 show the cases considered in this study: the horizontally layer case (fig. 1) which allows to analyze a grounding grid embedded in a soil with variations in the conductivity with depth, and the vertically layer case (fig.2) which allows to analyze the effect of changes in the conductivity in surroundings of the substation site. The statement of the latter soil model case and the derivation of its corresponding kernel $k_{b c}\left(\boldsymbol{x}_{c}, \boldsymbol{\xi}\right)$ differs slightly from the more common former one and it can be found in [13].

For this kind of soil models, integral kernels can be expressed in the general form:

$$
k_{b c}\left(\boldsymbol{x}_{c}, \boldsymbol{\xi}\right)=\sum_{l=0}^{l_{k}} k_{b c}^{l}\left(\boldsymbol{x}_{c}, \boldsymbol{\xi}\right), \quad k_{b c}^{l}\left(\boldsymbol{x}_{c}, \boldsymbol{\xi}\right)=\frac{\psi^{l}(\kappa)}{r\left(\boldsymbol{x}_{c}, \boldsymbol{\xi}^{l}(\boldsymbol{\xi})\right)},
$$

where $\psi^{l}$ is a weighting coefficient that only depends on a certain ratio $\left(\kappa=\left(\gamma_{1}-\gamma_{2}\right) /\left(\gamma_{1}+\gamma_{2}\right)\right)$ defined in terms of the conductivities of the layers, $r\left(\boldsymbol{x}_{c}, \boldsymbol{\xi}^{l}(\boldsymbol{\xi})\right)$ is the Euclidean distance between the points $\boldsymbol{x}_{c}$ and $\boldsymbol{\xi}^{l}$, being $\boldsymbol{\xi}^{0}$ the point $\boldsymbol{\xi}$ on the electrode surface $\left(\boldsymbol{\xi}^{0}(\boldsymbol{\xi})=\boldsymbol{\xi}\right)$ and $\boldsymbol{\xi}^{l}(l \neq 0)$ the images of $\boldsymbol{\xi}$ with respect to the earth surface and with respect to the interfaces between layers [11]. The number of summands $\left(l_{k}\right)$ in the integral kernel series depends on the case (e.g., $l_{k}=2$ for the uniform soil model, $l_{k}=4$ for the vertically layered soil model, $l_{k}=\infty$ for the horizontally layered soil model). Explicit expressions for these kernels can be found in $[12,13]$. 


\subsection{Variational Form of the Problem}

Expression (3) is essential for the solution of the problem, since it provides a way of computing the potential at any point of the ground in terms of the leakage current density that flows from the surface of the electrodes. Therefore, obtaining this leakage current density is the key of the problem, since it allows to obtain all the safety and design parameters of the grounding system when a fault current is derived through the earthing grid [9].

The unknown leakage current density $\sigma$ can be obtained by taking into account that integral expression (3) is also satisfied on the electrode surface $\Gamma$, where potential value is given by the boundary condition $V_{b}(\boldsymbol{\chi})=1, \forall \boldsymbol{\chi} \in \Gamma$. Consequently, $\sigma$ must satisfy the Fredholm integral equation of the first kind on $\Gamma$

$$
\frac{1}{4 \pi \gamma_{b}} \iint_{\boldsymbol{\xi} \in \Gamma} k_{b b}(\boldsymbol{\chi}, \boldsymbol{\xi}) \sigma(\boldsymbol{\xi}) d \Gamma=1, \quad \forall \boldsymbol{\chi} \in \Gamma .
$$

Now, imposing that the above equation is verified in the sense of weighted residuals, the following integral identity

$$
\iint_{\boldsymbol{\chi} \in \Gamma} w(\boldsymbol{\chi})\left(\frac{1}{4 \pi \gamma_{b}} \iint_{\boldsymbol{\xi} \in \Gamma} k_{b b}(\boldsymbol{\chi}, \boldsymbol{\xi}) \sigma(\boldsymbol{\xi}) d \Gamma-1\right) d \Gamma=0,
$$

must hold for all members $w(\boldsymbol{\chi})$ of a suitable class of test functions defined on $\Gamma[7,9]$. Clearly, a numerical formulation based on the Boundary Element Method is the best choice to solve equation (6), since it should only require discretization of the boundary, that is, the surface of the cylindrical conductors.

\section{BEM Numerical Solution}

The starting point for solving integral equation 6 is the discretization of the electrode surface $\Gamma$, and the unknown leakage current density $\sigma$. Thus, for given sets of $\mathcal{N}$ trial functions $\left\{N_{i}(\boldsymbol{\xi})\right\}$ defined on $\Gamma$ and $\mathcal{M} 2 \mathrm{D}$ boundary elements $\left\{\Gamma^{\alpha}\right\}$, we obtain

$$
\sigma(\boldsymbol{\xi})=\sum_{i=1}^{\mathcal{N}} \sigma_{i} N_{i}(\boldsymbol{\xi}), \quad \Gamma=\bigcup_{\alpha=1}^{\mathcal{M}} \Gamma^{\alpha}
$$

Thus, we can derive the discretizated expression

$$
\begin{gathered}
V_{c}\left(\boldsymbol{x}_{c}\right)=\sum_{i=1}^{\mathcal{N}} \sigma_{i} V_{c, i}\left(\boldsymbol{x}_{c}\right), \quad V_{c, i}\left(\boldsymbol{x}_{c}\right)=\sum_{\alpha=1}^{\mathcal{M}} \sum_{l=0}^{l_{V}} V_{c, i}^{\alpha l}\left(\boldsymbol{x}_{c}\right) \\
V_{c, i}^{\alpha l}\left(\boldsymbol{x}_{c}\right)=\frac{1}{4 \pi \gamma_{b}} \iint_{\boldsymbol{\xi} \in \Gamma^{\alpha}} k_{b c}^{l}\left(\boldsymbol{x}_{c}, \boldsymbol{\xi}\right) N_{i}(\boldsymbol{\xi}) d \Gamma^{\alpha}
\end{gathered}
$$

where $l_{V}$ represents the number of summands considered in the kernel series evaluation, being $l_{V}=l_{k}$ if the latter is finite, or the necessary number of terms to consider otherwise until convergence is achieved.

Finally, for a given set of $\mathcal{N}$ test functions $\left\{w_{j}(\boldsymbol{\chi})\right\}$ defined on $\Gamma$, variational form (6) is reduced to the linear system of equations

$$
\sum_{i=1}^{\mathcal{N}}\left[\sum_{\beta=1}^{\mathcal{M}} \sum_{\alpha=1}^{\mathcal{M}} \sum_{l=0}^{l_{R}} R_{j i}^{\beta \alpha l}\right] \sigma_{i}=\left[\sum_{\beta=1}^{\mathcal{M}} \nu_{j}^{\beta}\right], \quad j=1, \ldots, \mathcal{N}
$$

being

$$
\begin{gathered}
R_{j i}^{\beta \alpha l}=\frac{1}{4 \pi \gamma_{b}} \iint_{\boldsymbol{\chi} \in \Gamma^{\beta}} w_{j}(\boldsymbol{\chi}) \iint_{\boldsymbol{\xi} \in \Gamma^{\alpha}} k_{b b}^{l}(\boldsymbol{\chi}, \boldsymbol{\xi}) N_{i}(\boldsymbol{\xi}) d \Gamma^{\alpha} d \Gamma^{\beta}, \\
\nu_{j}^{\beta}=\iint_{\boldsymbol{\chi} \in \Gamma^{\beta}} w_{j}(\boldsymbol{\chi}) d \Gamma^{\beta}
\end{gathered}
$$

where $l_{R}$ represents the number of summands considered in the kernel series evaluation, being $l_{R}=l_{k}$ if the latter is finite, or the necessary number of terms to consider otherwise until convergence is achieved.

Resolution of linear system (10) gives the values of the unknowns $\sigma_{i}(i=1, \ldots, \mathcal{N})$, that allow to compute the current density leaking from the grid. However, this approach implies an unaffordable computing effort in CPU time when it 
is applied to the grounding analysis in practical cases. The fact is that the discretization of a 2D domain (the whole surface $\Gamma$ of the grounded electrodes) is required. This implies a large number of degrees of freedom. Moreover, the matrix of the system is full and the computation of its coefficients requires to integrate twice on $2 \mathrm{D}$ domains. We conclude that it is necessary to introduce some additional hypotheses in order to decrease the computational cost [9]. This leads to an approximated BEM formulation. Since complete derivation of the latter is lengthy [9, 11], we will only expose its highlights.

The key point of the approximated formulation is the circumferential uniformity hypothesis of the leakage current density in the cylindrical conductors of the grounding grid. Thus, we assum that the leakage current density $\sigma$ is constant around the cross section of the cylindrical conductors. Therefore, discretizations (7) and (10) become much simpler since the classes of test and trial functions are restricted to those with circumferential uniformity, and only the axial lines of the grounding electrodes have to be discretized [9]. In comparison with the general 2D approach, the number of elemental terms (11) and (12) needed to state linear system (10) and the number of unknowns $\sigma_{i}$ are significantly smaller.

Despite this important reduction in the computing cost extensive computing is still necessary, mainly because of the circumferential integration on the electrodes that is involved in the integral kernels. In previous works we have proposed the evaluation of these circumferential integrals in an approximated form by using specific quadratures, and we have derived a highly efficient analytical procedure to evaluate the coefficients of the linear system of equations in the case of uniform soil models [9]. Now, since the approximated expressions for elemental coefficients (9) and (11) are formally equivalent to a series of those obtained in the case of uniform soil, their computation can also be performed analytically [11]. Finally, it it important to remark that the proposed Boundary Element formulation is a general theoretical framework which includes, among others, the computer methods currently used in grounding analysis [6]. Furthermore, more accurate approaches can now be derived with different selection of sets of trial and test functions in the numerical scheme[9, 11]. All in all, the proposed numerical approach is a powerful, versatile and efficient method for earthing analysis which allows to obtain highly reliable and accurate results.

\section{Examples and Discussion}

The proposed BEM numerical approach has been implemented in a CAD system for earthing analysis. In this section we will present results obtained by using different kinds of soil models in the analysis of a real grounding grid: the Santiago substation, close to the city of Santiago de Compostela in Spain. The earthing system of this substation is a grid of 534 cylindrical conductors (diameter: $11.28 \mathrm{~mm}$ ) buried to a depth of $75 \mathrm{~cm}$, supplemented with 24 vertical rods (each one has a length of $4 \mathrm{~m}$ and a diameter of $15.0 \mathrm{~mm}$ ). The grounding system, which is given in figure 4 , protects a total area of $38,000 \mathrm{~m}^{2}$. The studied area is a wider superimposed rectangular zone of $320 \times 280 \mathrm{~m}^{2}\left(\right.$ i.e., $90,000 \mathrm{~m}^{2}$ approximately). The Ground Potential Rise (GPR) considered in this study is $10 \mathrm{kV}$.

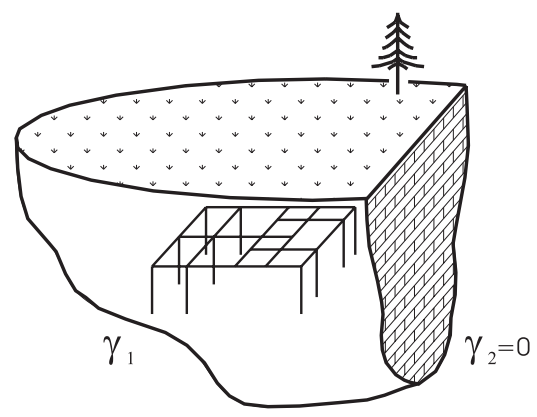

Figure 3. Scheme of the soil model considered in case D.

The numerical model used in the resolution of this problem is based on a Galerkin type weighting. Each bar is discretized in one single linear leakage current density element, which implies a total of 386 degrees of freedom (for this case, the use of one single constant density element per electrode would imply a total of 582, while the use of one single parabolic element would imply 968).

We have analyzed four cases in the Santiago grounding system for different kinds of soil models. "Model A" corresponds to an uniform soil model with $\gamma=40^{-1}(\Omega \mathrm{m})^{-1}$. "Model B" corresponds to an horizontally layered soil model (fig. 1) with $\gamma_{1}=40^{-1}(\Omega \mathrm{m})^{-1}, \gamma_{2}=400^{-1}(\Omega \mathrm{m})^{-1}$, being $1.6 \mathrm{~m}$ the thickness of the upper layer. "Model C" corresponds to a 


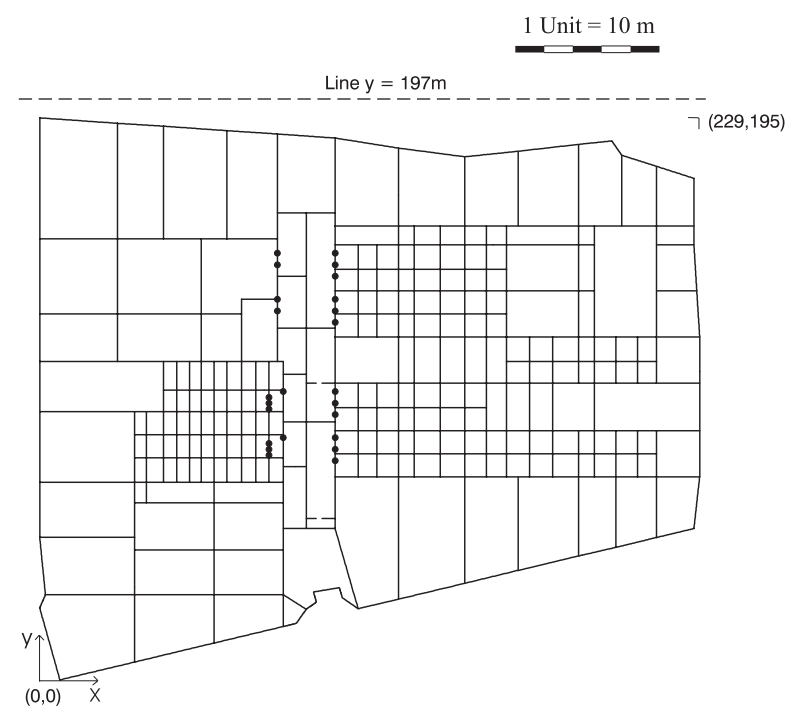

Figure 4. Santiago grounding system: Plan of the earthing grid (the situation of the ground rods is marked with black points).

Table 1. Numerical results for the different soil models

\begin{tabular}{|l|r|r|r|r|}
\hline & \multicolumn{4}{|c|}{ Soil Models } \\
\cline { 2 - 5 } & $\mathrm{A}$ & $\mathrm{B}$ & $\mathrm{C}$ & $\mathrm{D}$ \\
\hline Equiv. Resistance $(\Omega)$ & 0.0985 & 0.758 & 0.127 & 0.132 \\
Total Current $(\mathrm{kA})$ & 101.5 & 13.19 & 79.03 & 75.55 \\
CPU Time & $19 "$ & 112 & $36 "$ & $36 "$ \\
\hline
\end{tabular}

vertically layered soil model (fig. 2) with $\gamma_{1}=40^{-1}(\Omega \mathrm{m})^{-1}, \gamma_{2}=400^{-1}(\Omega \mathrm{m})^{-1}$, where the vertical interphase is at a distance of $2 \mathrm{~m}$ from the grounding grid (line $\mathrm{y}=197 \mathrm{~m}$ in the plan of figure 4 ). "Model D" corresponds to a soil model like model $\mathrm{C}$ with $\gamma_{1}=40^{-1}(\Omega \mathrm{m})^{-1}, \gamma_{2}=0(\Omega \mathrm{m})^{-1}$, where the vertical interphase is to $2 \mathrm{~m}$ far from the grounding grid. Soil model D would correspond to a very common situation in practice in the case of electrical substations located near urban zones when the vertical interphase is a retaining wall (fig. 3).

The numerical approach has been implemented on a CAD system, which has been compiled and run onto an Origin 2000 Silicon Graphics computer at the European Center for Parallelism of Barcelona, CEPBA (although the O2000 is a high-performance computer with 64 MIPS R10000 processors at $250 \mathrm{MHz}$, all the examples presented in this paper have been solved in sequential mode). Table 1 shows the results obtained for each case: the equivalent resistance of the grounding system, the total current derived to the soil, and the CPU time. Figures 5, 6, 7 and 8 show the potential distribution on the earth surface obtained by using the different soil models when the grounding electrode attains the GPR voltage. It is obvious that the plotted potential distributions differ significantly. However, it is known that noticeably different contour line drawings do not necessarily correspond to significant differences in the results. For this reason, in figure 9 we compare the potential profiles computed along different lines on the ground surface.

These examples show that the use of more complex soil models than the homogeneous one produces remarkably different results. Therefore, if one wishes to obtain accurate potential distributions and thus safer designs of grounding systems, soil should be modelled with reasonably faithful geometries and conductivities to the underlying reality. As an example, we can see that the existence of a retaining wall in model D produces noticeably different results in comparison with those obtained with model $\mathrm{C}$, since the expected rise of the potential level can be observed in fig 8 . We conclude that using these kinds of multi-layer soil models could be advisable to analyze grounding systems as a general rule, in spite of the increase in the computational effort. In fact, the use of this kind of advanced models should be mandatory in cases where the conductivity of the soil changes markedly with depth or in the vicinity of the substation site.

Obviously, this boundary element formulation can be applied to any other case with a higher number of layers. However, CPU time may increase up to an unacceptable level, mainly due to the poor rate of convergence of the underlying series expansions.

Nowadays, single-layer models run in real-time in conventional computers for the analysis of medium/large size grounding 


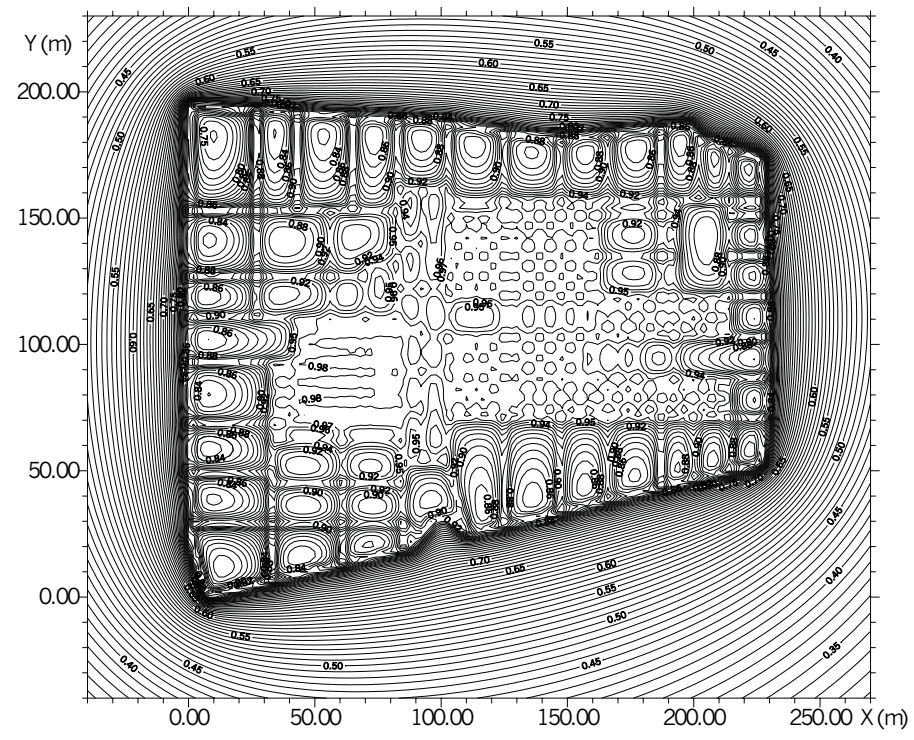

Figure 5. Potential distribution $(\times 10 \mathrm{kV})$ on earth surface (model A)

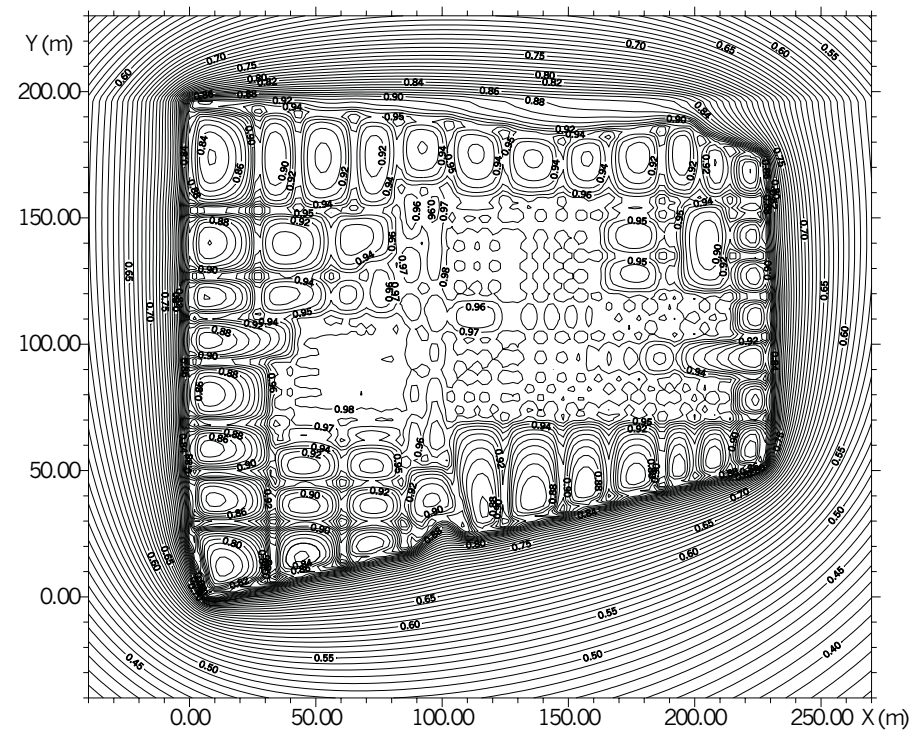

Figure 6. Potential distribution $(\times 10 \mathrm{kV})$ on earth surface $($ model B)

grids [9, 10], while multiple-layer models require an out of order computing time as a general rule. For this reason, we are studying the possible improvement of the convergence rate of the kernel series by using extrapolation techniques. On the other hand, we also working in the parallelization of the multi-layer boundary element numerical approach, which could become a real-time design tool for grounding analysis whenever this kind of supercomputing resources will be widespread available [14].

\section{Conclusions}

The numerical formulation developed by the authors for grounding analysis in uniform soils has shown to be highly effective. Onto this basis, we have proposed a generalization for more complex soil models, which allows to analyze the influence of the variations in the soil conductivity with depth and in the surroundings of the grounding system.

The suitable selection of the soil model is a key point in grounding analysis. We have shown that it is possible to obtain highly accurate results with the proposed method, and these results can be noticeably different depending on the kind of soil model considered. In short, the presented numerical technique is a powerful and efficient method that has been implemented in a CAD system for grounding analysis. This system allows to obtain both, quick results for a basic uniform soil analysis in real time, as much as more accurate results for a more detailed multilayer analysis. 


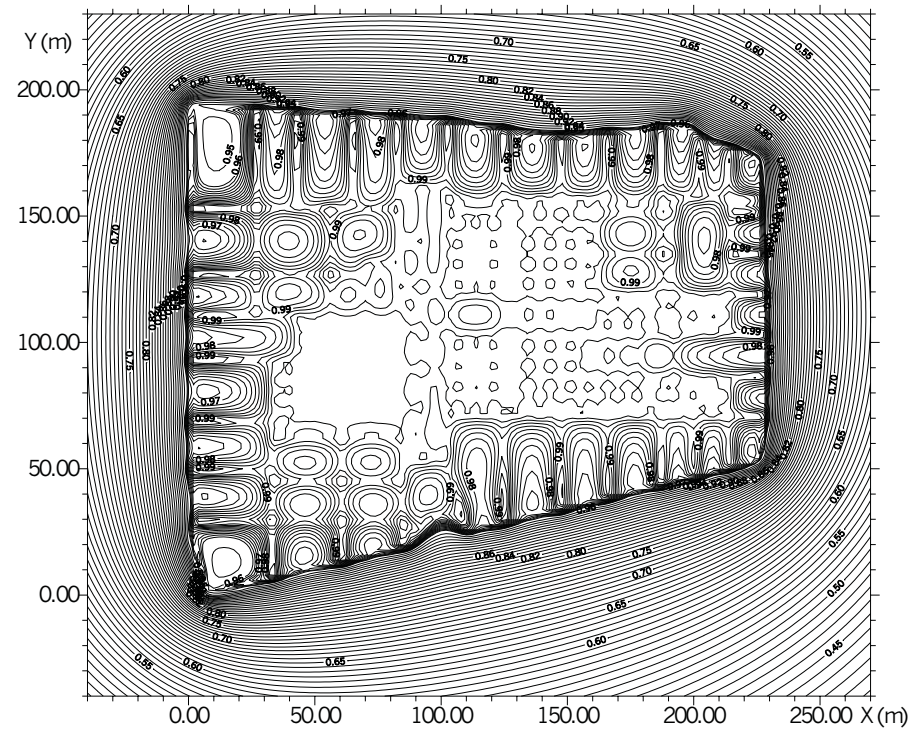

Figure 7. Potential distribution $(\times 10 \mathrm{kV})$ on earth surface (model $\mathrm{C}$ )

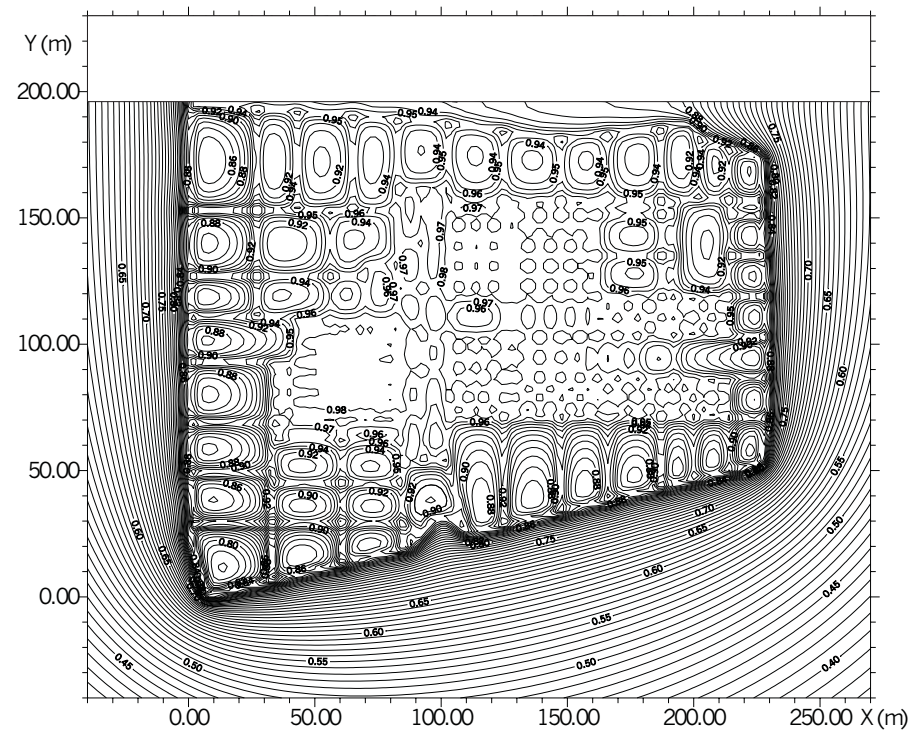

Figure 8. Potential distribution $(\times 10 \mathrm{kV})$ on earth surface $($ model $\mathrm{D})$

\section{Acknowledgements}

This work has been partially supported by the "Ministerio de Ciencia y Tecnología (project \#1FD97-0108)" of the Spanish Government, cofinanced with European Union FEDER funds, by the power company "Unión Fenosa Ingeniería S.A.", and by research fellowships of the "European Center for Parallelism of Barcelona, CEPBA", the Autonomous Government "Xunta de Galicia" and the "Universidad de La Coruña".

\section{References}

[1] ANSI/IEEE Std.80, Guide for safety in AC substation grounding, IEEE, New York, (2000).

[2] J.G. Sverak et al., Safe substation grounding, Part I: IEEE Transactions on Power Apparatus and Systems, 100, 4281-4290, (1981); Part II: IEEE Transactions on Power Apparatus and Systems, 101, 4006-4023, (1982)

[3] J.G. Sverak, Progress in step and touch voltage equations of ANSI/IEEE Std 80. Historical perspective, IEEE Transactions on Power Delivery, 13, 762-767, (1998). 


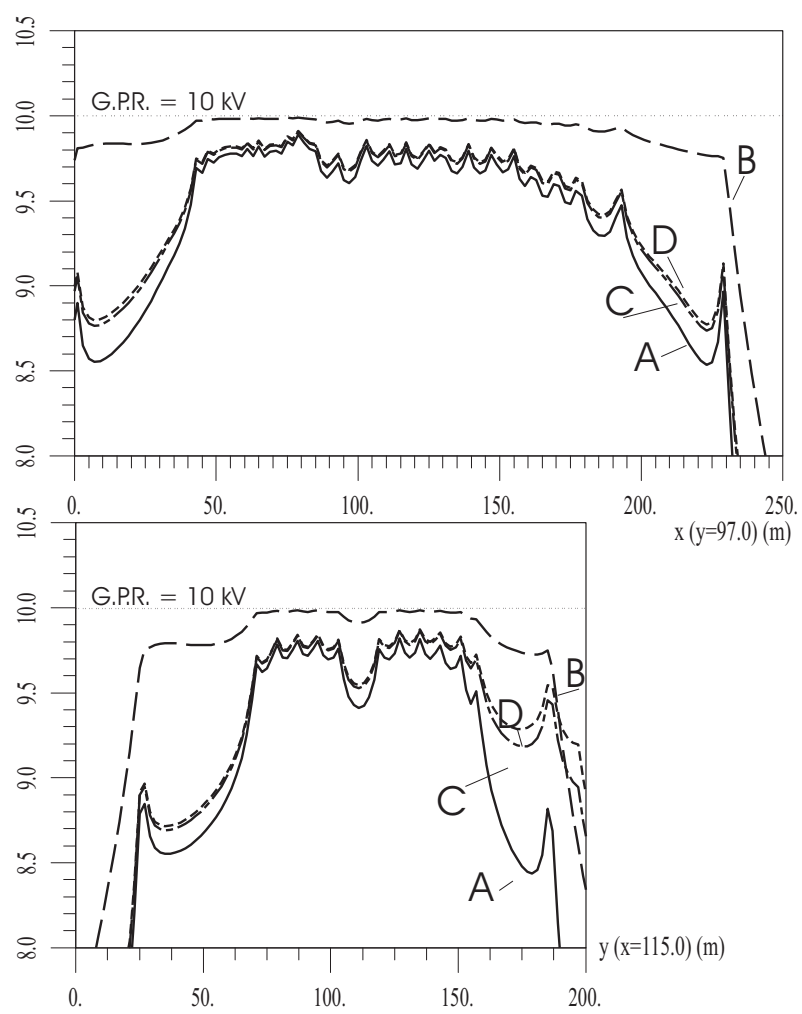

Figure 9. Potential profiles $(\mathrm{kV})$ on the earth surface along two lines.

[4] R.J. Heppe, Computation of potential at surface above an energized grid or other electrode, allowing for non-uniform current distribution, IEEE Transactions on Power Apparatus and Systems, 98, 1978-1988, (1979).

[5] D.L. Garrett, J.G. Pruitt, Problems encountered with the Average Potential Method of analyzing substation grounding systems, IEEE Transactions on Power Apparatus and Systems, 104, 3586-3596, (1985).

[6] F. Navarrina, I. Colominas, M. Casteleiro, Why do computer methods for grounding analysis produce anomalous results?, Submitted to IEEE Transactions on Power Delivery, (2000).

[7] F. Navarrina, I. Colominas, M. Casteleiro, Analytical integration techniques for earthing grid computation by BEM, In "Numerical Methods in Engineering and Applied Sciences", 1197-1206, CIMNE Pub., Barcelona, Spain (1992).

[8] I. Colominas, A CAD system for grounding grids in electrical installations: A numerical approach based on the Boundary Element Integral Method (in spanish), Ph.D. Thesis, Universidad de La Coruña, Spain, (1995).

[9] I. Colominas, F. Navarrina, M. Casteleiro, A boundary element numerical approach for grounding grid computation, Computer Methods in Applied Mechanics and Engineering, 174, 73-90, (1999).

[10] I. Colominas, F. Navarrina, M. Casteleiro, A boundary element formulation for the substation grounding design, Advances in Engineering Software, 30, 603-700, (1999).

[11] I. Colominas, F. Navarrina, M. Casteleiro, A numerical formulation for grounding analysis in stratified soils, Submitted to IEEE Transactions on Power Delivery, (1999).

[12] G.F. Tagg, Earth resistances, Pitman, New York (1964).

[13] I. Colominas, J. Gómez-Calviño, F. Navarrina, M. Casteleiro, A general numerical model for grounding analysis in layered soils, In "Computational Civil and Structural Engineering", 209-216; Civil-Comp Press, Edinburgh, UK (2000).

[14] I. Colominas, F. Navarrina, G. Mosqueira, J.M. Eiroa, M. Casteleiro, Numerical modelling for grounding grids in high-performace parallel computers, In "Boundary Elements XXII", WIT Press., Southampton, UK (2000). 